\title{
Traitement de vases de l'estuaire de la Seime
}

\author{
Jean-Pierre Troalen \\ maitre de conférences à l'INPL, LG, Ecole Nationale Supérieure de Géologie \\ Catherine Duron et Céline Hagnéré \\ élèves-ingénieurs ENSG, LG
}

\begin{abstract}
Résumé:
Il est nécessaire avant tout stockage, tout transport terrestre ou fluviatile, de modifier l'état physique initial des vases. Une fois réalisée l'identification physique et chimique initiale des vases, nous avons relié ces dọnnées aux paramètres résultant de la modification - à l'aide de différents traitemerîts - de cet état physique initial. Les résultats obtenus, par traitement au ciment $C F J$ et par traitement avec un "super-absorbant", montrent une évolution des propriétés physiques et mécaniques, en particulier un abaissement de la teneur en eau et un accroissement de la cohésion. Le comportement de ce nouveau matériau dépend de la teneur en eau initiale des vases, de la teneur en ciment ou de super-absorbant et, en conséquence, de la structure acquise.
\end{abstract}

\section{Introduction}

Modifier l'état physique des vases issues du dragage avant tout stockage, tout transport terrestre ou fluviatile, apparait comme la première des nécessités en raison de leur caractère thixotropique. Ces vases apparaissent comme des complexes soleau essentiellement variables dans le temps et possédant des propriétés très différentes de celles de leurs constituants élémentaires, particulièrement en raison de la présence de matières organiques. Nous avons donc axé notre intervention sur les paramètres riécessaires à la compréhension du processus de modification des structures et des propriétés surfaciques des vases. En première approche, nous avons utilisé deux types de traitements : du ciment CPJ et un produit "superabsorbant". L'application de ces traitements demande beaucoup de précautions et des connaissances élémentaires préalables.

\section{Caractérisation des vases}

Les différents essais ont été réalisés (Duron et Hagnéré, 1995) sur des carottes de vases de Seine provenant de l'avant-port du Havre et prélevées directement par fonçage de tubes PVC. Cette opération de prélévement a été effectuée par le LMFGC de l'Université du Havre et les échantillons étaient a priori de même nature que ceux utilisés par Marot (1994). A l'ouverture des carottes, il était possible de noter macroscopiquement, une zone bioturbée de 0 à $40 \mathrm{~cm}$ de profondeur correspondant à une vase de couleur claire, puis une vase noire sur le reste de la longueur. 


\subsection{Identification physique et chimique}

Selon la norme NF-P-94.050, les résultats obtenus sur l'ensemble des échantillons montrent que les valeurs moyennes des teneurs en eau pondérale permettent de distinguer quantitativement les deux domaines de vases observés à l'ouverture :

$$
1 \mathrm{H}: 93,4 \%<\mathrm{w} \%<133,2 \% \quad 2 \mathrm{H}: 140,2 \%<\mathrm{w} \%<168,3 \%
$$

Cette variabilité entre les valeurs de la teneur en eau peut sans doute être expliquée par les hypothèses de différences d'état physique initial, de granulométrie et de composition minéralogique, qu'il conviendra de vérifier.

Selon la norme NF:P-18.558, le poids volumique absolu est défini comme étant le quotient de la masse sèche de l'échantillon par le volume de sa matière tous vides déduits. La variabilité des valeurs moyennes du poids volumique absolu entre les deux domaines de vases apparait encore nettement :

$$
1 \mathrm{H}: \gamma_{\mathrm{S}}=25,3 \mathrm{kN} / \mathrm{m}^{3} . \quad 2 \mathrm{H}: \gamma_{\mathrm{S}}=21,4 \mathrm{kN} / \mathrm{m}^{3}
$$

Par la méthode dite de l'essai "à la tâche" (NF-P-94.068), les résultats montrent une différence assez peu significative entre les valeurs au bleu moyennes obtenues sur les deux domaines de vases:

$$
1 \mathrm{H}: \mathrm{VB}=2,39 \quad 2 \mathrm{H}: \mathrm{VB}=2,45
$$

Par la méthode du calcimètre Bernard, les valeurs des pourcentages en $\mathrm{CaCO}_{3}$ obtenus sur les deux domaines de vases présentent, cette fois encore, une faible variabilité :

$$
1 \mathrm{H}: 30,20 \%<\mathrm{CaCO}_{3} \%<32,08 \quad 2 \mathrm{H}: 29,89 \%<\mathrm{CaCO}_{3} \%<30,09
$$

En raison de la faible variabilité des valeurs au bleu et du pourcentage en carbonate de calcium, il n'a pas été tenu compte pour la suite des essais de la distinction entre les deux domaines de vases observés. En ce sens, nous rejoignons le cas d'un matériau dragué et, en ce sens, "homogénéisé" par cette opération.

Selon la norme NF-P-94.051 (limites d'Atterberg) et sur la totalité des échantillons des deux domaines de vases, la valeur moyenne de la limite de liquidité est de $79,6 \%$ et celle de la limite de plasticité est égale à $44,1 \%$. L'indice de plasticité moyen est de $35,5 \%$. En prenant une valeur moyenne de $107,7 \%$ pour la teneur en eau naturelle, l'indice de consistance est alors égal à - 0,79 et, dans le diagramme de plasticité de Casagrande, ces vases apparaissent comme des limons très plastiques.

La composition minéralogique des vases a été obtenue par diffractométrie des rayons $x$ et donne, pour les 5 premiers éléments minéraux ( $63 \%$ de l'échantillon total), la répartition suivante :
quartz $: 33 \%$
calcite : $25 \%$
pyrite : $1 \%$
feidspaths : $3 \%$

L'analyse spécifique de la fraction dimensionnelle inférieure à $2 \mu \mathrm{m}$ (37\%) fait apparaître la composition suivante en minéraux argileux s.s. : montmorillonite : $20 \%$ kaolinite : $10 \%$ illite : $7 \%$

\subsection{Aralyses dimensionnelles}

Pour des raisons intéressant le développement ultérieur de notre recherche, nous avons réalisé l'analyse dimensionnelle des vases par trois méthodes de mesure distinctes dont les principes different totalement. Signalons ici que la quasi totalité 
des échantillons possédait des dimensions inférieures à $80 \mu \mathrm{m}$ et qu'il était possible, dans ce cas, de négliger la fraction supérieure à $80 \mu \mathrm{m}$.

\subsubsection{Sédimentométrie}

Selon la norme NF-P-94.057, cette méthode classique est basée sur la loi de Stokes et utilise un densimètre. En considérant la fraction inférieure à $2 \mu \mathrm{m}$ comme celle correspondant au pourcentage en "argiles $G$ " ( $G$ au sens granulomètrique), nous obtenons une valeur en argiles $G$ comprise entre $26 \%$ et $22 \%$ pour l'ensemble des échantillons testés.

\subsubsection{Sédigraph 5000}

Cet appareil mesure par l'intermédiaire d'un faisceau de rayons $x$ la concentration des particules qui restent en suspension à une hauteur de sédimentation diminuant en fonction du temps. La vitesse de sédimentation suit comme précédemment la loi de Stokes. Les courbes de sédimentation, obtenues sur l'ensemble des échantillons de vases, donnent pour la fraction inférieure à $2 \mu \mathrm{m}$, une valeur du pourcentage en argiles $\mathrm{G}$ comprise entre $30 \%$ et $21 \%$. C'est-à-dire un intervalle de valeurs proche de celui obtenu par sédimentomètrie.

\subsubsection{Malvern Mastersizer}

Cet appareillage utilise les propriétés de diffraction de la lumière (faisceau laser) par les particules solides en suspension dans un fluide. L'angle de diffraction de la lumière est alors inversement proportionnel à la taille des particules. Sur l'ensemble des échantillons de vases testés, le pourcentage de la fraction inférieure à $2 \mu \mathrm{m}$ reste très proche de $10 \%$, ce qui pose nettement le problème de corrélation avec les deux méthodes d'analyse dimensionnelle précédentes.

\subsubsection{Commentaires sur l'analyse dimensionnelle}

Les trois méthodes utilisées, bien qu'elles considérent chacune une forme sphérique pour les particules constituantes, ne sont pas basées sur les mêmes lois et donneront des résultats d'autant plus justes que le matériau étudié contiendra des particules de forme sphérique, ce qui semble a priori ne pas être le cas des sédiments très fins que sont les vases de Seine et les vases en général (Troalen, 1994). De plus, ces trois méthodes s'appliquent à des domaines d'analyse différents (>1 $\mathrm{\mu m}$ pour la sédimentométrie ; de 0,1 à $100 \mu \mathrm{m}$ pour le Sédigraph 5000 ; de 0,1 à $600 \mu \mathrm{m}$ pour le Malvern Mastersizer) et, la quantité de matériau utilisé pour l'analyse est elle même variable $(80 \mathrm{~g}$ pour la sédimentométrie, $4 \mathrm{~g}$ pour le Sédigraph, $1 \mathrm{~g}$ pour le Malvern Mastersizer). Ce qui va poser d'entrée le problème de ta représentativité de l'échantillon à analyser. Cette comparaison entre méthodes doit faire l'objet d'une étude bien plus approfondie, surtout si nous voulons comparer ces résultats à ceux donnés par l'analyse minéralogique. Cependant; il est possible d'utiliser comme paramètres indicateurs les "diamètres équivalents". (dimensions des particules correspondant aux pourcentages cumulés de 10,50 et $90 \%$ ), c'est ce qui a été réalisé sur le tableau 1 suivant :

\begin{tabular}{|llll|}
\hline Méthode & $d_{10}(\mu \mathrm{m})$ & $\mathrm{d}_{50}(\mu \mathrm{m})$ & $\mathrm{dg}_{0}(\mu \mathrm{m})$ \\
\hline sédimento & 0,2 & 5 & 40 \\
\hline Sédigraph & 0,9 & 13 & 26 \\
\hline Malvern & 2,9 & 15 & 47 \\
\hline
\end{tabular}




\section{Caractérisation mécanique et traitement}

La caractérisation mécanique a principalement porté sur la cohésion (ou résistance au cisaillement) des vases intactes et remaniées. L'évolution de la cohésion a été ensuite suivie sur ces mêmes vases après traitement, soit par du ciment CPJ, soit par un super-absorbant.

NB : Le terme intact s'applique aussi bien aux échantillons témoins avant la première mesure de cohésion au scissomètre $\left(C_{\text {int }}\right)$ qu'à la première mesure de cohésion sur les échantillons de vase traités au ciment et au super-absorbant. Les termes de cohésion résiduelle et de cohésion remaniée conservent ensuite le sens défini par la norme de l'essai au scissomètre.

\subsection{Cohésion ou résistance au cisaillement}

Les mesures de la cohésion ont été réalisées à l'aide d'un scissomètre de laboratoire, selon la norme NF-P-94.012. Il s'agit ici des mesures de cohésion effectuées directement sur les carottes de vases (cohésion intacte et cohésion résiduelle) et sur les prélévements réalisés aussitôt après (cohésion remaniée). Ces résultats figurent sur le tableau 2 ci-après et concernent les deux domaines de vases différenciées macroscopiquement lors de l'ouverture des carottes.

\begin{tabular}{|cc|}
\hline $1 \mathrm{H}$ (cohésion, $\mathrm{kPa}$ ) & $2 \mathrm{H}$ (cohésion, $\mathrm{kPa})$ \\
\hline $2,05<\mathrm{C}_{\text {int }}<6,05$ & $1,64<\mathrm{C}_{\text {int }}<2,77$ \\
\hline $1,44<\mathrm{C}_{\text {rés }}<1,74$ & $0,41<\mathrm{C}_{\text {rés }}<1,85$ \\
\hline $0,61<\mathrm{C}_{\text {rem }}<1,33$ & $0,21<\mathrm{C}_{\text {rem }}<1,03$ \\
\hline
\end{tabular}

Tableau $2:$ mesure de la cohésion sur les vases intactes

Les valeurs qui figurent sur le tableau 3, concernent les mesures de cohésion réalisées sur les deux mêmes domaines de vases, mais après remaniement et homogénéisation de l'ensemble des matériaux correspondants. Nous nous plaçons ici dans le cas d'un mélange analogue à celui issu d'un dragage. Cette analogie est possible en raison des teneurs en eau initiales élevées.

\begin{tabular}{|cc|}
\hline $1 \mathrm{H}($ cohésion, $\mathrm{kPa})$ & $2 \mathrm{H}$ (cohésion, $\mathrm{kPa})$ \\
\hline $0,72<\mathrm{C}_{\text {int }}<1,23$ & $0,82<\mathrm{C}_{\text {int }}<1,23$ \\
\hline $0,51<\mathrm{C}_{\text {rés }}<1,03$ & $0,72<\mathrm{C}_{\text {rés }}<1,13$ \\
\hline
\end{tabular}

Tableau $3:$ mesure de la cohésion des vases remaniées

La variabilité des valeurs de la cohésion des vases remaniées apparaît bien entendue comme plus faible que celle observée pour les vases intactes. D'une façon générale, les valeurs de la cohésion des vases intactes et celles des vases remaniées doivent être considérées comme le reflet de comportements distincts de vases dont la structure a été modifiée par les remaniements successifs. La variabilité des valeurs de la cohésion entre les deux domaines de vases intactes devrait pouvoir être interprétée au regard des teneurs en eau initiales plus élevées dans la série $2 \mathrm{H}$ (tableau 2). Or, cette interprétation ne peut être uniquement validée que pour les seules valeurs de la cohésion intacte des vases dans leur état initial naturel. La valeur maximale de la cohésion résiduelle de la série $2 \mathrm{H}$ pose un problème, lequel est encore plus accentué dans le cas des vases remaniées puisque les valeurs des cohésions intactes et résiduelles de la série $2 \mathrm{H}$ peuvent être supérieures à celles de la série $1 \mathrm{H}$, à linverse de ce qui se passe dans les vases naturelles intactes. 
Il est sans doute possible d'interpréter ces données par l'étude de l'évolution des structures, mais la solution viendra certainement de l'étude des matières organiques contenues (Troalen, 1994, 1995) puisque celles-ci jouent un rôle important dans l'organisation et les liaisons interparticulaires qui caractérisent la structure.

\subsection{Amélioration des propriétés mécaniques}

Les deux traitements proposés ont été réalisés sur l'ensemble des vases de Seine prélevées après malaxage et homogénéisation des échantillons. L'état physique initial des vases avant leurs traitements respectifs sera donc a priori considéré comme identique è il est alors possible, par. la mesure des paramètres spécifiques de cet état physique, d'en suivre l'évolution à l'issue du traitement. La mesure de l'évolution de ces mêmes paramètres en fonction du temps est relativement difficile en raison des vitesses de réaction de prise du mélange, surtout dans le cas de l'utilisation du produit super-absorbant.

\subsubsection{Procédure d'essai}

Après prélévement, malaxage et homogénisation des vases, il a été procédé à un stockage provisoire destiné à maintenir les échantillons dans le même état physique initial. Ensuite, pour chaque essai, il est procédé à la mesure de la cohésion de l'échantillon témoin, puis à la mesure de la cohésion de l'échantillon immédiatement après son traitement. La procédure de traitement a été rigoureusement la même dans les deux cas. Prélévement d'une masse définie de vase, pesée et ajout de 1,2 et $3 \%$ de la masse initiale humide en ciment ou en super-absorbant. Cet ajout s'effectue par simple soupoudrage (ciment ou super-absorbant sous forme de poudre anhydre), suivi d'un malaxage du matériau réalisé directement dans le récipient les contenant et destiné à la mesure de la cohésion qui est aussitôt pratiquée.

\subsubsection{Traitement au ciment CPJ}

Le mélange vase-ciment a été réalisé selon des pourcentages de 1,2 et $3 \%$ par rapport à la masse initiale humide. Pour l'ensemble des échantillons traités, la valeur de la teneur en eau finale est nettement inférieure à celle de la teneur en eau initiale. La valeur au bleu diminue en fonction de l'élévation du pourcentage de ciment et correspond à une diminution de la surface spécifique accessible : genèse d'agrégats au sein de la vase et structure de moins en moins lâche. Ce comportement est validé par les valeurs de la porosité et de l'indice des vides qui diminuent ainsi que par la légère augmentation de la masse volumique absolue (tableau 4). La valeur de la cohésion intacte augmente en fonction du temps mais se stabilise assez vite, mais il est nécessaire d'atteindre $3 \%$ de ciment pour que cet accroissement soit significatif d'une amélioration réelle des propriétés mécaniques. L'évolution de la cohésion en fonction du temps de prise montre que celle-ci.croit très rapidement dans le cas du mélange à $3 \%$ de ciment et la stabilisation est atteinte au bout de 5 à 6 minutes (figure 1). La valeur de la cohésion intacte passe ainsi de $0,8 \mathrm{kPa}$ (témoin) à près de $1,5 \mathrm{kPa}$ (3\% ; les teneurs en eau passent, parallèlement, de $143 \%$ (initiale) à $131 \%$ (finale). La valeur de stabilisation de la cohésion peut être interprétée comme le seuil optimal d'un mélange donné, c'est-à-dire qu'elle représente le rapport optimum entre la quantité de ciment ajoutée et la quantité d'eau disponible dans le matériau. Ce rapport est naturellement influencé par la nature de - la structure, elle-même dépendante du rapport eau contenue / pourcentage et nature des matières organiques. Les matières organiques, notamment les gels, sont l'un des processus majeurs de la formation des liens interparticulaires de la structure. Et, il est donc nécessaire de "briser" au maximum ces liens lors de l'ajout du ciment et 
du malaxage qui lui fait suite. Cette accessibilité au ciment de la structure et de l'eau contenue va donc conditionner en grande part l'efficacité du traitementet la création de la nouvelle structure (Troalen, 1995).

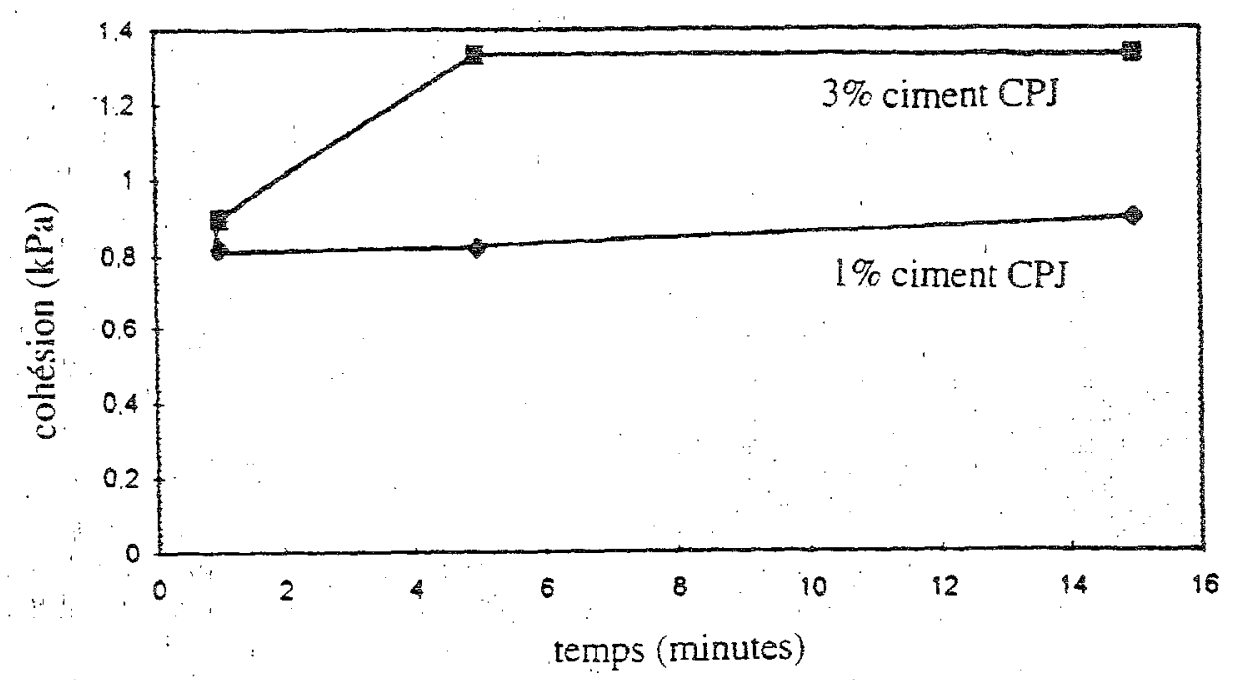

Figure 1 : Evolution de la cohésion intacte en fonction du temps. Cas des vases traitêes avec du ciment CPJ. (série $1: 1 \%$; série $2: 3 \%$ ).

\begin{tabular}{|c|c|c|c|c|c|c|}
\hline paramètres & traitem & ent cim & & traitem & ent supe & ar-absorbant \\
\hline & $1 \%$ & $2 \%$ & $3 \%$ & $1 \%$ & $2 \%$ & $3 \%$ \\
\hline$w_{\text {init }}(\%)$ & 143 & & & 143 & & \\
\hline$w_{\text {final }}(\%)$ & 138 & 135 & 131 & 137 & 129 & 125 \\
\hline $\mathrm{C}_{\text {init }}(\mathrm{kPa})$ & 0,84 & & & 0,84 & & $\therefore$ \\
\hline $\mathrm{C}_{\text {final }}(\mathrm{kPa})$ & 1,25 & 1,43 & 1,49 & 1,45 & 2,46 & 2,25 \\
\hline$\gamma_{\text {s init }}(g / \mathrm{cmin})$ & 2.52 & & & 2,52 & & : \\
\hline$\gamma_{\text {s final }}(\mathrm{g} / \mathrm{cm} 3)$ & 2,57 & 2,58 & 2,59 & 2,49 & 2,41 & 2,44 \\
\hline$n_{\text {init }}(\%)$ & 78,6 & & & 78,6 & & \\
\hline nfinal (\%) & 78,5 & 78,1 & 77,7 & 78,0 & 76,7 & 77,6 \\
\hline e & 3,668 & & & 3,668 & & \\
\hline efinal & 3,655 & 3,570 & 3,500 & 3,560 & 3,295 & 3,461 \\
\hline$\sqrt{B}$ init & 4,38 & & & 4,38 & & . \\
\hline$\sqrt{B}$ finale & 3,64 & 3,58 & 3,73 & 0,57 & 0,57 & 0,57 \\
\hline$S_{r}$ initial $(\%)$ & 98,8 & & & 98,8 & & \\
\hline Sr final $(\%)$ & 97,9 & 98,4 & 97,1 & 96,6 & 95,3 & 89,1 \\
\hline
\end{tabular}

Tableau 5 : comparaison entre les paramètres physiques et mécaniques des vases de Seine après traitement au ciment CPJ et au super-absorbant.

( $w$ = teneur en eau $; C=$ cohésion ; $\gamma_{S}=$ masse volumique absolue $; n=$ porosité ; $\mathrm{e}=$ indice des vides $; \mathrm{VB}=$ valeur au bleu $; \mathrm{Sr}=$ degré de saturation) 


\subsubsection{Traitement au super-absorbant}

Les mêmés échantillons de vases ont été traités cette fois par un produit superabsorbant (brevet G.T.I.Process) se présentant sous la forme d'une poudre très fine. Lies mêmés pourcentages que pour les mélanges avec le ciment ont été adoptés et la méthode de préparation du mélange est la même. Les mêmes paramètres ont été mesurés et sont reportés sur le tableau 4 . L'évolution de la cohésion est très brutale et le mélange acquijert très rapidement une certaine rigidité. La différence principale entre le mélange vase / super-absorbant et celui vase / ciment tient dans les processus de réaction de prise. En effet et même si dans les deux cas la teneur initiale joue une rôle important, la réaction de prise du süper-absorbant avec les vases se traduit par une sorte de "polymérisation à froid" et la création d'un réseau de liens qui résulte de la réaction entre le super-absorbant, l'eau contenue et la phase organo-minérale des vases. Cette réaction est d'autant plus importante que les vases seront plus riches en carbonate de calcium (Troalen, 1994, 1995). Il en résuite un comportement particulier des vases traitées et la-mesure des paramètres devient délicate y compris celle de la cohésion. C"est notamment le cas de la mesure de la teneur en eau finale puisqu'une part de l'eau initiale va être utilisée par la réaction de prise avec le super-absorbant (comme dans le cas du ciment) et que d'autre part, une fraction de l'eau initiale va se trouver piégée dans le réseau structural créé ; elle ne sera pas "libérée" par le passage néiessaire à l'étuve $105^{\circ} \mathrm{C}$ pour la mesure de la teneur en eau finale ; ce qui ne se produisait pas dans le cas du mélange vase-ciment. Nous parlerons donc dans ce cas de la teneur en eau finale apparente. Si les paramètres de porosité et d'indice des vides peuvent être mesurés, ils suivent d'ailleurs une évolution à peu près analogue à celle observée dans le cas du mélange vase-ciment, la valeur au bleu et la valeur de la cohésion.finale deviennent difficiles à saisir. Le réseau qui se crée par le mélange vase-superabsorbant empèche toute dispersion du matériau et, en conséquence, il ne peut y avoir fixation du bleu de méthylène (surfaces spécifiques globalement non accessibles).

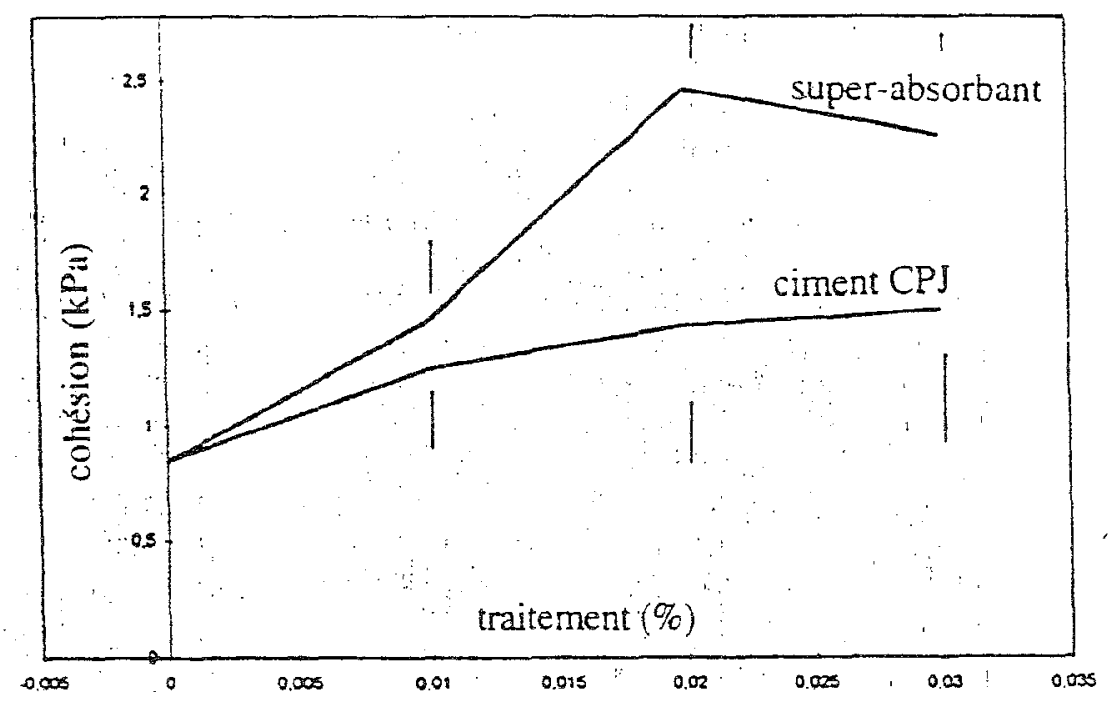

Figure 2 : Evolution de la cohésion en fonction du traitement 
De même, en raison de la prise très rapide du mélange, l'évolution de la cohésion dans le temps est difficile à mesurer. Malgré tout, nous n'avons pas observé de stabilisation de la valeur de la cohésion (figure 2) mais au contraire une décroissance de cette valeur (dès les $2 \%$ atteints). Ce qui est remarquable dans le cas d'un mélange vase / super-absorbant, c'eșt l'accroissement pratiquement immédiat de la cohésion, mais cette augmentation de la valeur de la cohésion n'est réelle que pour un pourcentage de super-absorbant compris entre 1 et $2 \%$. Dès le seuil de $2 \%$ dépassé, l'homogénéisation du mélange par malaxage devient impossible, là réaction de prise est trop rapide et les agrégats créés ne sont pas liés entre eux, d'où la chute de la valeur de la cohésion. Bien entendu, tout comme dans le cas du mélange vase / ciment, le type de structure et la teneur en eau initiale influencent la réaction de prise ; cette influence sera d'autant plus importante que la teneur en matières organiques sera initialement élevée.

\section{Conclusion}

Les deux traitements réalisés sur des échantillons de vases de l'estuaire de la Seine ont abouti à une modification immédiate de l'état physique des vases. La cohésión croît très rapidement en fonction de la diminution apparente de la teneur en eau. Nous constatons que la valeur de la cohésion est fonction du pourcentage de produit ajouté, mais que passé une certaine valeur de ce pourcentage, la cohésion n'évolue plus. L'existence d'un seuil à partir duquel les propriétés de la vase traitée se stabilisent est facilement mise'en évidence dans le cas du ciment CPJ. Par contre, dans le cas de l'utilisation du super-absorbant, le seuil se traduit cette fois par une valeur limite à partir de laquelle les propriétés mécaniques de la vase chutent rapidement. Ce comportement est à relier aux processus de prise qui sont fonction de la teneur en eau initiale, de la nature minéralogique et granulomètrique des constituants solides, ainsi que l'importance du pourcentage initial en matières organiques. Ces paramètres conditionnent la structure des vases et, en conséquence, leur accessibilité aux produits traitants et les propriétés mécaniques résultantes de ce nouveau matériau.

\section{Références bibliographiques}

Duron C., Hagnéré C., (1995), "Caractérisation et traitement de vases d'estuaire", Projet lle 2ème année, ENSG Nancy, 70.p.

Marot F., (1994), "Caractérisation et traitement des boues portuaires", Mémoire de DEA Génie Civil, Universités de Nantes et du Havre, BRGM, Ecole Centrale de Nantes, $104 \mathrm{p}$.

Troalen J.P., (1994), "A propos d'une nouvelle approche des vases et des boues d'estuaire : caractếrisation et devenir", 3èmes Journées Nationales Génie CôtierGéniẹ Civil, Sète (France), 2-4 mars 1994.

Troalen J.P., (1995), "Modifications des propriétés physico-mécaniques et st 4 cturales de vases d'estuaire par mélange avec un super-absorbant", Revue Canadienne de Géotechnique (à paraitre). 